\title{
Research and application of pressure decreasing technology of polymer material injection wells
}

\author{
Yang Taiwei ${ }^{1}$, Yan Yu ${ }^{1, *}$, Guo Shuzhao ${ }^{1}$, Cai Qingqin ${ }^{1}$, Yang Yang $^{1}$, Du Yuqiao ${ }^{1}$ \\ ${ }^{1}$ Petroleum Engineering Research Institute of Dagang Oilfield, Tianjin 300280
}

\begin{abstract}
Injecting polymer materials is an important means to improve the ultimate recovery, maintain stable production and improve the development effect of the oil field in the high water cut period. However, in the development process of polymer flooding, with the continuous increase of the cumulative polymer injection, the problem of high-pressure under-injection frequently occurs in the injection well. In this paper, based on geological characteristics and plugging material analysis, the causes of high pressure/under-injection and production characteristics of different types of measure wells. To study and optimize the rubber breaking, degradation and synergistic acidification liquid system that are applicable to different blockage conditions can effectively improve the adaptability of the blockage liquid system; Meanwhile, the formation of a quick plugging process with the characteristic of "single-stage single-step injection + prescription formula structure" can effectively improve the success rate of polymer plugging process measures, providing strong technical support for polymer flooding technology.
\end{abstract}

\section{Introduction}

To effectively guarantee the stable increase of the old oilfield production, the industrialized application of the tertiary oil recovery technology taking polymer flooding as the main body was conducted in Daqing, Shengli and other oil fields ${ }^{[1-2]}$. With the continuous increase of the cumulative polymer injection, the problem of highpressure \& under-injection occurred frequently in the injection well, which greatly affected on the development effect of the tertiary oil recovery. There were more than 260 polymer injection wells in D Oilfield. With the continuous development of polymer injection, there were different under-injection problems in each block. For injection wells with under-injection at high pressure due to polymer plugging, the strong oxidant system corrodes the pipe string and reacts easily with flammable gas, which poses construction risks, the validity period of nonoxidation plugging solution measure is short, and biological technology measures have disadvantages such as strong selectivity of conditions, so the construction safety should be guaranteed, and a variety of methods such as flowback plugging, deconstructing agent plugging, acidification and augmented injection which were practiced in the early stage have poor reproducibility. Therefore, it is urgent to improve the pertinence of the technology and develop effective deplugging measures.

\section{Analysis of reservoir characteristics}

The polymer injection area of D Oilfield was mainly heavy oil reservoir, which was mainly distributed in two layers: Minghua and Guantao, with shallow burial, high porosity and permeability, large pore-throat radius, relatively loose cementation, and high muddy content. A small part was located in Kongdian, with relative deep burial and small pore-throat radius.

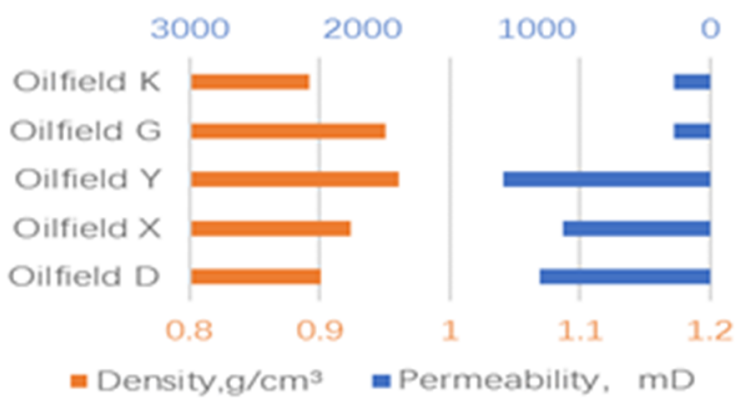

Fig. 1. Permeability and crude oil physical property statistics

\section{Research on plugging mechanism}

Through the investigation and the study on the polymer micelles returned from the well, the analysis thought that the main reasons for blockage were as follows ${ }^{[3]}$ :

\subsection{Influence of injection water quality on blockage}

The injected water was treated by precipitation, filtration, sterilization, deoxygenation and insolation, and then the water that met the water quality requirements was injected into the well through the surface pipe network. In the whole process, the wellhead of the injection well was

* Corresponding author: dg_yanyu@petrochina.com.cn 
prone to have substandard water quality, which was specifically reflected in too high solid impurity content, too high ion content and bacteria. Therefore, these conditions were easy to cause the combined action of polymer and iron hydroxide and ferrous sulfide, thus forming a blockage.

Table 1. Polymer micelles returned energy spectrum analysis

\begin{tabular}{|c|c|}
\hline Element & Content,\% \\
\hline $\mathrm{F}$ & 4.2 \\
\hline $\mathrm{O}$ & 50.9 \\
\hline $\mathrm{Ca}$ & 20.2 \\
\hline $\mathrm{Na}$ & 11.8 \\
\hline $\mathrm{Mg}$ & 7.6 \\
\hline $\mathrm{Fe}$ & 5.2 \\
\hline
\end{tabular}

\subsection{Impact of the polymer itself on blockage}

The polymer used in polymer flooding was a macromolecule industrial product, which contained certain impurities ${ }^{[4]}$. When the mechanical impurities contained in it were too high and incompatible with the formation properties, or the polymer solution denatured under formation conditions, the stratum would be blocked to a certain extent. Meanwhile, there were also risks in the injection process of polymer products, such as incomplete particle dissolution, adsorption, and hydraulic retention, which further decreased the size of the small channels and increased the flow resistance of the fluid ${ }^{[5]}$.

\subsection{Impact of physical properties of stratum on blockage}

The higher salinity of formation water would lead to the decrease of polymer solution viscosity and residual resistance coefficient ${ }^{[6]}$. What was more serious was that high valent metal ions would cause polymer crosslinking.
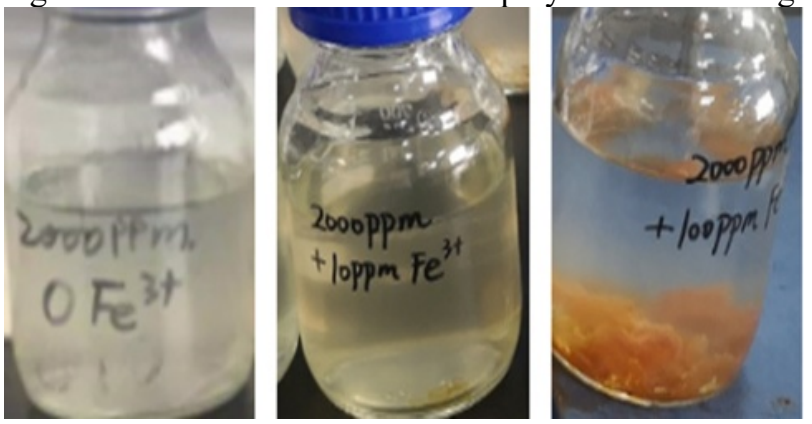

Fig. 2. Compatibility test of injected water and iron ion

\section{Analysis of production characteristics}

By combining with plugging mechanism research and block material analysis results, and through observing the production curve of under-injection well in high permeability blocks, the analysts thought that the injection allocation was completed stably in current injection pressure, and the injection well with decreased injection was recovered after stopping the injection. The reason for the high-pressure under-injection problem was the polymer crosslinking caused by free metal ions in the wellbore, eventually blocked the reservoir, and decreased the injection.

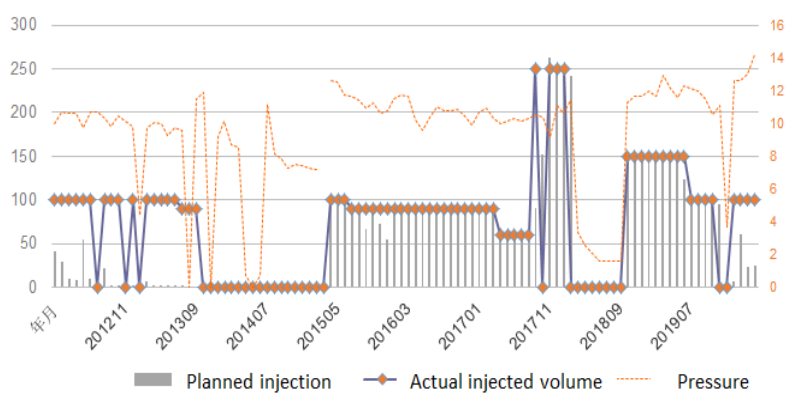

Fig. 3. Production characteristics of Well A

Under the current injection pressure, it was difficult to complete the injection allocation continuously and stably. For the injection well with gradually decreased injection capacity, the analysis showed that the reasons for the highpressure under-injection were crude oil emulsification, particle migration, asphaltene adsorption and deposition, etc., making the liquid absorption capacity of the dominant connected layers gradually decrease.

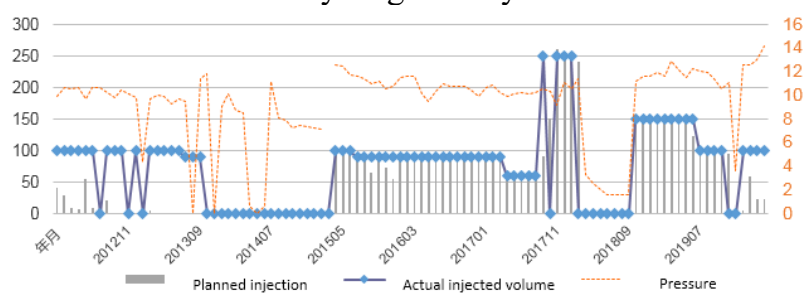

Fig. 4. Production characteristics of Well B

\section{Preferred measures of liquid system}

The further analysis of the returned micelles thought that, for the blocked polymer injection wells, metal ions made hydrophobic associated polymer systems have a crosslinking, intensified particle migration in the reservoir, and further coerced the mechanical impurities, sands, and precipitations to absorb crude oil, and gradually formed high-strength micelles with stable chemical properties to block the reservoir. Therefore, the degradation $\&$ acidified collaborative system should be selected.

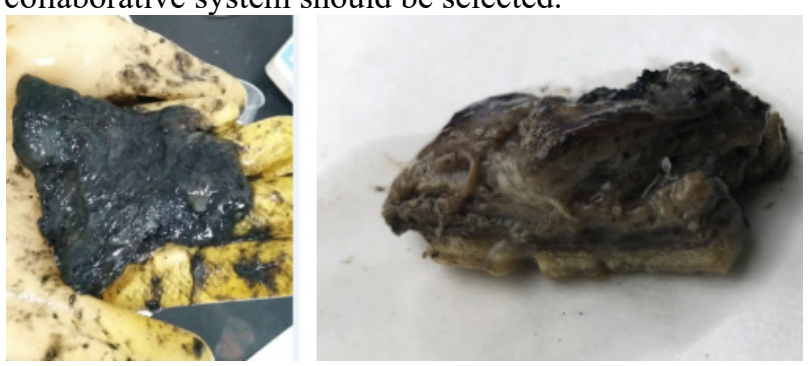

Fig. 5. The returned micelles for the polymer injection wells 


\subsection{Low-cost deplugging system in wellbore and area near the wellbore}

The under-injection of polymer injection well is primarily caused by polymer plugging. When the permeability ratio of the core exceeds $1000 \times 10^{-3} \mu^{2}$ and the concentration of sample with fouling is $0.1 \mathrm{mg} / \mathrm{L}$, the permeability loss as a result of polymer adsorption and retention is up to $20 \%-30 \%$. As proved by several practices made in the initial stage, any unplugging technology with a single function is incompatible with complex types of plugging. Moreover, the technologies associated with polymer unplugging are not fault-free, leading to unobvious outcome of unplugging and a short period of validity.

Due to the polymer crosslinking blockage caused by free metal ions, a low-cost deplugging system was selected in the wellbore and the area near the wellbore to degrade the gel breaking crosslinking polymers, while the iron ions were complexed and stabilized, and a selfassembled membrane was formed on the surface of the string to reduce the impact of metal ions in the wellbore.
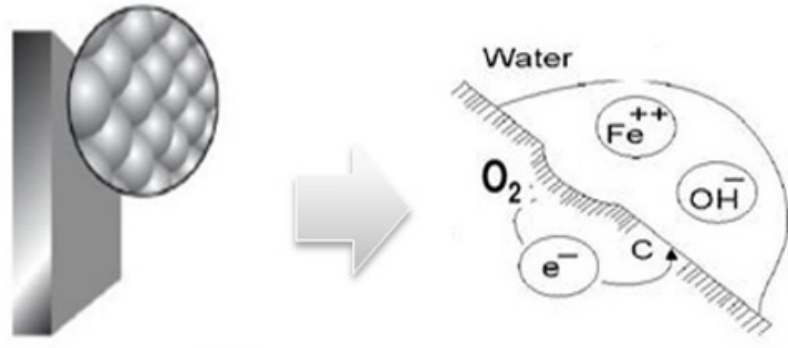

Fig. 6. Schematic diagram of deplugging system complexed and stabilized the iron ions

= Degradation rate, $\%$ = Price, Yuan $/ \mathrm{t}$

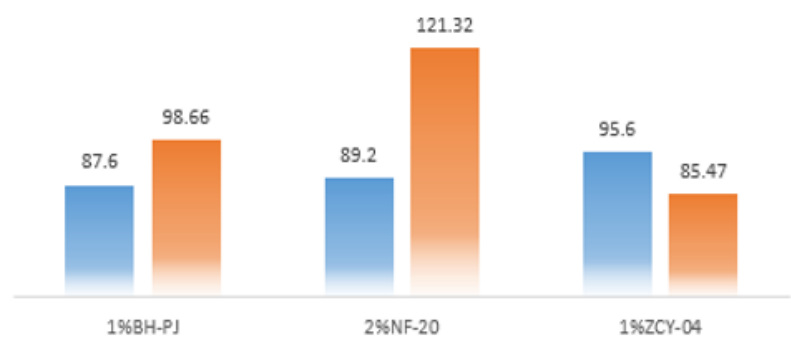

Fig. 7. The experiment of low-cost deplugging system

\subsection{Low damage inorganic debugging liquid system}

Several sampling results showed that there was a big difference of water quality between water injection station and ending injection pipeline. An amount of suspended solids and inorganic impurities were found by the very end of the injection pipeline. Acid wash may remove the impurities, but the disadvantages are also obvious: high cost of treatments, long working period and especially for the injection of wells.

Sandstone reservoir is usually acidized with hydrochloric acid and hydrofluoric acid. Hydrofluoric acid can simply react with siliceous or carbonate rocks in the matrix, but its reaction with silicates such as clay or feldspar tends to be highly complex. Hydrofluoric acid can react with most components (quartz, feldspar, clay, carbonate) in sandstone at different rates, the reaction products are likely to generate insoluble fluoride precipitates that may clog up the pore throat. Regular acidizing process is performed on the low permeable reservoir, most of the acid active substances are consumed in the vicinity of wellbore.

Under any condition, Fluoboric acid does not contain a large amount of hydrofluoric acid, which means it has low reactivity. The hydrofluoric acid in fluoroboric acid solution will produce many hydrofluoric acids by hydrolysis when consumed. Therefore, its total dissolving power is comparative to mud acid. Fluoboric acid can be used as a pre-flush before the treatment with mud acid (sensitivity formation), which can avoid particle instability and subsequent pore plugging; alternatively, it can be used as a subject acid for sandstone matrix or the formation which contains many clay minerals.

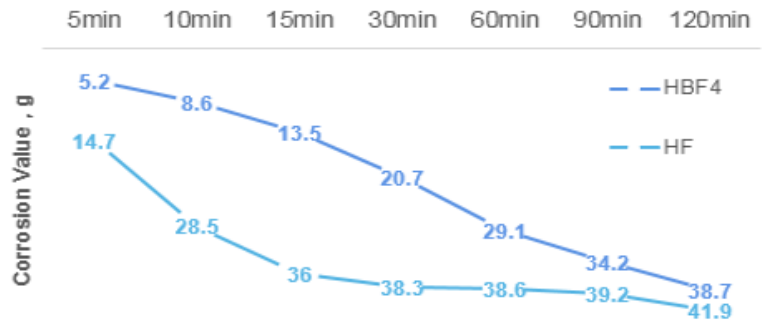

Fig. 8. Comparison of the dissolution ability of fluoroboric acid and hydrofluoric acid

To solve the blockage of mechanical impurities, clay particle migration and asphaltine adsorption and deposition, the low damage inorganic deplugging liquid system was selected. The dissolution ability of inorganic scale was up to $92.6 \%$, the dissolution rate of sandstone was $48.2 \%$, and the reaction rate was $1 / 3 \sim 1 / 4$ of that of the soil acid, which not only effectively increased the treatment radius of acid solution but also effectively prevented particle migration.

\section{aHydrochloric acid =Mudacid a AR-mud acid}

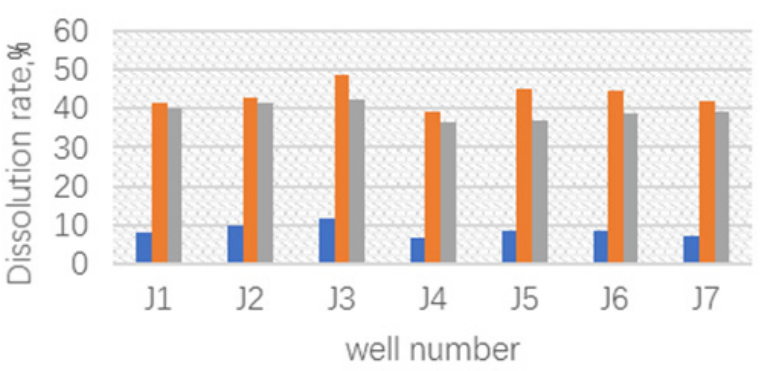

Fig.9. Evaluation on dissolving power of acidic fluid system

\section{Optimization of polymer deplugging technology}

There is an interlayer pressure difference in the 
waterflooding development of heterogeneous reservoir. When the bottom-hole pressures applied on two layers are identical, the pressure of high permeable layer rises at a higher rate than that of low permeable layer in the vicinity of the bottom-hole zone. As a result, there is an interlayer pressure difference in the vicinity of the bottom-hole zone. According to the theoretical calculation, the interlayer pressure difference is up to $100 \mathrm{~kg} / \mathrm{cm}^{2}$ when the difference of layer-to-layer permeability is 10 times.

The single-stage fluid system was used to simplify the injection process and the tank bettery preparation, in view of the different reformation needs of the separate injection wells and the conventional plugging needs such as metal ion crosslinking, scaling and plugging, residual oil deposition/emulsification in the wellbore and nearwellbore area. Then by compounding the existing additives, the addition was conducted to the complex blockage, while the subtraction was conducted to the simple blockage, thus forming a "prescription" formula structure; Later, through the differential strategy of cleaning and dredging the superior channels and corroding and linking up the inferior channels, the single-system directional deplugging of the general injection well and the balanced transformation of separate injection wells were realized.

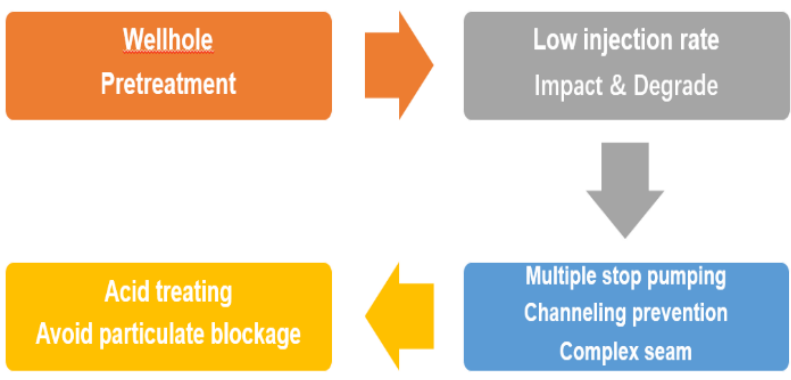

Fig. 10. Construction process flow of Optimization of polymer deplugging technology

Optimization of polymer deplugging technology for polymer injection wells exploits the cooperative action of polymer unplugging agent and acid liquid to get the wellbore fouling completely treated. Degrading liquid is used to unplug wellbore and the seepage channel in the vicinity of wellbore. With continuous injection at a low rate and multiple pumping stops, this measure can degrade polymer and dissolve polymer micelles. In the meanwhile, the Polymer coating for rock and mechanical impurities is stripped off. In doing so, acid liquid can fully dissolve the exposed sand and clay minerals, expand the pore throat, unplug carbonate and silicate minerals, and avoid reservoir plugging and secondary packing due to subsequent particle migration, allowing for deep and longstanding chemical unplugging.

\section{Practice and application conclusion}

The synergistic deplugging technology of polymer degradation and acidification was applied in Well X1 of D Oilfield, which was is an injection well with high porosity and high permeability. In the early stage, the injection was stopped many times due to drilling utilization, and the daily injection volume decreased after the resumption of injection.

The analysis showed that the well had an underinjection caused by the crosslinking in the wellbore, so the low-cost deplugging system was used in the wellbore and near-wellbore area, and the single-stage injection without moving the pipe string was used. After the implementation, the initial daily injection volume was $74 \mathrm{~m}^{3} / \mathrm{d}$.

Table 2. Application of Polymer deplugging technology

\begin{tabular}{|c|c|c|c|}
\hline \multirow{2}{*}{ No. } & \multirow{2}{*}{$\begin{array}{c}\text { Volume of } \\
\text { the Treating Fluid } \\
\left(\mathbf{m}^{\mathbf{3})}\right.\end{array}$} & \multicolumn{2}{|c|}{$\begin{array}{c}\text { Daily Injection Volume } \\
\left(\mathbf{m}^{\mathbf{3}} \mathbf{)}\right.\end{array}$} \\
\cline { 3 - 4 } & & Before & After \\
\hline Well X1 & 40 & 50 & 124 \\
\hline Well X2 & 40 & 5 & 48 \\
\hline Well X3 & 40 & 26 & 49 \\
\hline
\end{tabular}

Combined with the analysis of the reservoir and production characteristics, the technology of polymer material injection wells has been used in 3 Wells. It had a remarkable effect on the pressure decreasing and augmented injection of the polymer injection wells.

\section{References}

1. W.DeMing, C.JieCheng, W.JunZheng, etc, Acta Petrolei Sinica,2005,26(1)

2. Z.Li, Petroleum Exploration and Development, 2007,34(1)

3. W.YanPing, H.Hong, W.XiongXiong, etc,Ion Exchange and Adsorption,2013,29(6)

4. L.XingRong, T.Le, W.Lu, etc, Contemporary Chemical Industry,2017,46(6)

5. J.ZhaoHua, Fujian Chemical Industry,2015(8)

6. P.GuiQing, Inner Mongolia Petrochemical Industry ,2013(11) 